\title{
Tendências das Pesquisas de Gênero na Formação Docente em Ciências no Brasil
}

\section{Trends in Gender Research in Teacher Education in Science}

\author{
Amanda O. Proença, Matheus J. Baldaquim, Irinéa L. Batista e Fabiele C. D. Broietti
}

Resumo: Buscou-se, por meio de uma análise documental, esboçar um quadro a respeito das discussões de Gênero na pesquisa em Formação Docente e em Educação em Ciências presentes em dois eventos (ENPEC e ANPED), no Brasil. Para tal, foram analisados trabalhos publicados nas atas das edições de 2005 a 2017 dos eventos selecionados mediante busca nos resumos. Elaboraram-se Unidades de Registro prévias e as interpretações seguiram os princípios da Análise de Conteúdo. Evidenciou-se um crescimento de publicações nesta temática e o predomínio de alguns focos de pesquisa, tais como: questões de Gênero articuladas à prática docente; artigos de revisão teórica; participação feminina na História da Ciência; frequência relativa do Gênero feminino e suas relações com a Ciência; abordagens metodológicas articulando Gênero no ensino de Ciências; e questões de Gênero em diferentes mídias. Nesse sentido, ressaltamos a relevância da continuidade e aprofundamento de tais pesquisas na Formação Docente e na Educação Científica.

Palavras-chave: Gênero e Ciência. Educação Científica. Formação Docente.

\begin{abstract}
Searched through a documental analysis, to outline a framework regarding gender discussions in research in science teaching education present in two events (ENPEC and ANPED), in Brazil. For that, an analysis of published works in the proceedings of the editions from 2005 to 2017 the events selected through search in the abstracts. The previous Registration Units were elaborated, and the interpretations followed the Analysis of Content. There was an increase in the number of papers that deal with this topic and the predominance of some research centers. The papers was about gender issues articulated to teaching practice; theoretical review articles; the participation of women in the history of science; relative frequency of the female gender and its relations with science; methodological approaches to gender issues in science teaching; and gender issues in different media. In this sense, we emphasize the relevance of continues and deeper research in science teaching education as Scientific Education.
\end{abstract}

Keywords: Gender and Science. Scientific Education. Teacher Training.

\footnotetext{
Amanda Oliveira Proença (amanda.proenca@ @otmail.com) é licenciada em Química pela Universidade Tecnológica Federal do Paraná (UTFPR). Atualmente, é mestranda no Programa de Pós-Graduação em Ensino de Ciências e Educação Matemática da Universidade Estadual de Londrina (PECEM-UEL). Londrina, PR - BR. Matheus Junior Baldaquim (matheusbaldaquim@gmail.com) é licenciado em Química e especialista em Educação Matemática e Ciências pela UTFPR. Atualmente, é mestrando no Programa de Pós-Graduação em Educação para a Ciência e a Matemática da Universidade Estadual de Maringá (PCM-UEM). Maringá, PR - BR. Irinéa de Lourdes Batista (irinea2009@ gmail.com) é graduada em Física, mestre em Ensino de Ciências e doutora em Filosofia. Atualmente, é professora associada no Departamento de Física, e docente e orientadora no PECEM-UEL. Londrina, PR - BR. Fabiele Cristiane Dias Broietti (fabieledias@uel.br) é licenciada em Química, mestre e doutora em Ensino de Ciências. Atualmente, é professora adjunta no Departamento de Química, e docente e orientadora no PECEM-UEL. Londrina, $\mathrm{PR}-\mathrm{BR}$.
}

Recebido em 21/08/2018, aceito em 14/01/2019

A seção "Cadernos de Pesquisa" é um espaço dedicado exclusivamente para artigos inéditos (empíricos, de revisão ou teóricos) que apresentem profundidade teórico-metodológica, gerem conhecimentos novos para a área e contribuições para o avanço da pesquisa em Ensino de Química. 
A história da participação da mulher no âmbito social sempre foi marcada por lutas e batalhas que nem sempre foram favoráveis a elas, e na Ciência isso não é diferente. No início do século XIX, o papel feminino tinha valor no casamento para se alcançar a estabilidade financeira ou se encontrava como dama de companhia ou como professora de Educação Infantil. As áreas de engenharia, física, química e biologia raramente foram a principal escolha das mulheres. Alice Rossi (1965) atribui esse fato a incompatibilidades biológicas e/ou sociais, bem como a prioridade pelo casamento, pela maternidade, e pela interferência dos pais na formação profissional de seus filhos.

Segundo uma revisão de Matthews (1995), os estudos históricos abordam uma Ciência androcêntrica e machista derivada da Ciência ocidental. Tindall e Hamil (2004) discutem a afirmação de que comportamentos ativos relacionados a meninos e passivos relacionados a meninas influenciam na escolha da carreira profissional, o que gerou grande desinteresse feminino em pesquisas científicas e em estudos em instituições e universidades, quando se refere a Ciências aplicadas ou da Natureza. Esses dados são confirmados por Melo et al. (2004), que relatam que apesar da crescente discussão do papel feminino na sociedade, a presença de mulheres que cursam o Ensino Superior nas áreas de Ciências da Natureza e tecnológicas no Brasil ainda é muito pequena.

O destaque para as mulheres na Ciência veio com Mozans, em 1913, com o primeiro escrito a respeito da participação das mulheres na Ciência. No âmbito internacional, pesquisas (Harding e McGregor, 1996; Batista et al., 2011; 2013) mostram que a partir dos anos 70 houve um crescimento significativo das mulheres em instituições e universidades importantes na área das Ciências. Outras mulheres pesquisadoras se tornaram destaque quando se referem a estudos de Gênero na Ciência e Tecnologia, elas são: Evelyn Foz Keller, Sandra Harding, Donna Haraway, Margaret Rossiter, Sally Gregory Kohlstedt, Helen Longino e Londa Schiebinger.

O objetivo das discussões realizadas por essas mulheres na Ciência é apresentar a ausência da participação feminina, questionando a sub-representação na história da humanidade e as definições do que é Ciência, baseando-se nos estudos de Gêneros (Lopes, 1998).

A temática Gênero em pesquisas brasileiras ainda está em crescimento e grande parte das discussões tem sido na área da Educação e Ciências Sociais. Entretanto, como já relatado em Rezende e Ostermann (2007), na área de Ciências da Natureza em específico, ainda são deficientes as discussões relacionadas às questões de Gênero.

Em um levantamento realizado por Souza (2008, p. 151) não foram encontrados artigos que abordassem questões de Gênero "em duas das mais importantes publicações sobre Ensino e Ciências - Investigações em Ensino de Ciências (140 artigos) e Ciência \& Educação (216 artigos)" entre os anos de 1998 a 2007. Batista et al. (2011) realizaram um levantamento em atas de dois eventos da área de Ensino de Ciências e em periódicos da área de Ensino de Ciências e Educação Matemática no Brasil entre os anos 2005 a 2011 e foram encontrados apenas sete trabalhos ao total que abordavam questões de Gênero e Formação Docente.

No Brasil, atualmente, existe um grupo de estudos em Investigações em Filosofia e História da Ciência e Educação em Ciências e Matemática (IFHIECEM, 2019) na Universidade Estadual de Londrina que possui um projeto em desenvolvimento: "Produção científica feminina: sua estrutura e dinâmica e seu papel na Formação Docente". Este grupo está há oito anos pesquisando a respeito desta temática, com produções de teses, dissertações e publicações em periódicos e eventos da área.

A partir desses estudos, considera-se a necessidade de pesquisas científicas estarem cada vez mais próximas ao professor, dando destaque à formação histórico-epistemológica a respeito da participação feminina na História da Ciência. Desse modo, as discussões a respeito de Gênero e da participação feminina na Ciência podem ser inseridas no currículo e na Formação Docente, para que, então, o entendimento da Ciência se torne contextualizado e apresente a contribuição de pesquisas femininas ao longo da construção histórica.

Dando prosseguimento aos levantamentos já realizados por Batista et al. (2013) e Chiari e Batista (2015), este trabalho apresenta resultados de um levantamento realizado nos anos de 2015 a 2017 nas atas dos eventos da Associação Nacional de Pós-Graduação e Pesquisa em Educação (ANPED) e Encontro Nacional de Pesquisa em Educação em Ciências (ENPEC) a respeito da temática Gênero e formação de professores. O objetivo central consistiu em esboçar um quadro evidenciando aspectos e tendências das discussões de Gênero nas pesquisas em Formação Docente em Educação em Ciências no Brasil de 2005 a 2017.

\section{Procedimentos Metodológicos}

Este trabalho busca dar continuidade nos levantamentos realizados e publicados por membros do grupo de pesquisa Investigações em Filosofia e História da Ciência e Educação em Ciências e Matemática, em que Batista et al. (2011) realizaram um levantamento nos eventos ANPED e ENPEC, entre os anos de 2005 a 2011, e Chiari e Batista (2015) realizaram o levantamento nos mesmos eventos no período de 2010 a 2014. Neste artigo damos prosseguimento à investigação realizando o levantamento no período de 2015 a 2017, bem como uma análise metateórica desses dados.

Por seu impacto nacional na área de Ensino de Ciências e Matemática, os eventos analisados foram ANPED e ENPEC nos eixos temáticos Formação de Professores e Diversidade, Multiculturalismo e Educação em Ciências. Nesse contexto, foram consultadas as atas do ENPEC e ANPED no período de 2015 a 2017, pesquisando a temática 'Gênero' na Educação Científica e na Formação Docente em Ciências da Natureza.

Considerando os objetivos e a temática de investigação, 
optou-se pelo desenvolvimento de uma pesquisa qualitativa de cunho interpretativo, tendo como pressuposto metodológico a análise documental (Bogdan e Biklen, 1994). Para o tratamento e a interpretação dos dados coletados utilizou-se a análise de conteúdo proposta por Bardin (2004). Para a exploração do material foram utilizadas Unidades de Registro prévias com base no referencial teórico e nos objetivos da pesquisa.

\section{Levantamento Realizado em Atas de Eventos 2005-2014}

Nesta seção, são apresentadas as referências dos trabalhos identificados nos artigos de Batista et al. (2011) em que foram encontrados quatro trabalhos entre 2005 a 2011 e os seis trabalhos encontrados por Chiari e Batista (2015) entre os anos de 2010 a 2014 nos mesmos eventos. Os trabalhos encontrados, as referências e seus códigos estão destacados nos Quadros 1 e 2 apresentados a seguir.

\section{Levantamento Realizado em Atas de Eventos 2015-2017}

Por meio da busca realizada nas atas do ANPED e do ENPEC, de 2015 e 2017, foi possível encontrar 21 trabalhos que relacionavam Gênero na Educação Científica e a Formação de Professores. Sendo 15 apresentados no ENPEC e 6 apresentados no ANPED. Abaixo são apresentadas as referências dos trabalhos, o evento e o código de cada trabalho.

Pode-se perceber um aumento nos últimos anos na quantidade de trabalhos apresentados no ANPED e no ENPEC relacionados ao tema Gênero. No ano de 2013 encontrou-se apenas três publicações no evento ENPEC e na edição seguinte, em 2015, observa-se no Quadro 2 um total de seis artigos com a mesma temática, possibilitando novas discussões. Em 2017, destaca-se um crescimento com 9 trabalhos apresentados. Isso demonstra uma possibilidade de maior visibilidade ao tema no decorrer dos últimos anos.

Nota-se também o crescimento no número de trabalhos no evento ANPED, em que no ano de 2015 foram apresentados apenas dois trabalhos relacionados à temática pesquisada e dois anos depois o número de trabalhos foi quatro.

Outro fator perceptível são as publicações do grupo de pesquisa Investigações em Filosofia e História da Ciência e Educação em Ciências e Matemática, entre os anos de 2005 a 2014 foram apresentados apenas dois trabalhos, já nas últimas edições foram apresentados seis trabalhos com tal problematização.

\section{Exploração do Material: Construção das Unidades de Registro}

A seguir, são apresentadas as Unidades de Registro prévias (UR) para a análise dos artigos encontrados (atas de 2015 a 2017). Foram utilizadas as Unidades de Registros propostas por Batista et al. (2011) e por Silva et al. (2017):

UR 1. Gênero e materiais didáticos. Os artigos classificados nesse eixo analisam como ocorrem as representações de Gênero nos livros didáticos (Batista et al., 2011, p. 5).

UR 2. Gênero e inferências de interpretação. Os artigos nesse eixo estudam os motivos da frequência relativa do Gênero feminino e suas relações com a Ciência em cursos de nível médio, técnico e superior de ensino (Batista et al., 2011, p. 5).

Quadro 1: Trabalhos publicados em eventos nacionais entre 2005 e 2011

\begin{tabular}{|c|c|c|}
\hline Evento & Artigo/Autores & Código \\
\hline ANPED 2006 & $\begin{array}{l}\text { Educando as novas gerações: representações de gênero nos livros didáticos de matemática/CASAGRANDE, L. } \\
\text { S. e CARVALHO, M. G. }\end{array}$ & $\mathrm{T} 1$ \\
\hline ENPEC 2007 & $\begin{array}{l}\text { Uma análise das diferenças de gênero no discurso escolar/LIMA JÚNIOR, P. R. M.; OSTERMANN, F.; REZENDE, } \\
\text { F. e CAVALCANTI, C. J. H. }\end{array}$ & T2 \\
\hline ENPEC 2007 & $\begin{array}{l}\text { Visão de alunos sobre a predominância feminina no programa de vocação científica da Fundação Oswaldo Cruz/ } \\
\text { SOUSA, I. C. F.; BRAGA, C. N.; FRUTUOSO, T. M.; FERREIRA, C. A. e VARGAS, D. S. }\end{array}$ & T3 \\
\hline ENPEC 2009 & Gênero e educação científica: uma revisão da literatura/LIMA JÚNIOR, P. R. M.; OSTERMANN, F. e REZENDE, F. & T4 \\
\hline ENPEC 2011 & $\begin{array}{l}\text { Gênero feminino e formação de professores na pesquisa em educação científica e matemática no Brasil/BATISTA, I. } \\
\text { L.; TOREJANI, A. T. C.; HEERDT, B.; LUCAS, L. B.; OHIRA, M. A.; CORRÊA, M. L.; BARBOSA, R. G. e BASTOS, V. C. }\end{array}$ & T5 \\
\hline ENPEC 2011 & Questões de gênero e sexualidade na sala de aula: um relato dos professores/LIMA, A. C. L. M. e SIQUEIRA, V. H. F. & T6 \\
\hline ENPEC 2011 & $\begin{array}{l}\text { Possíveis relações entre HFC, concepção da natureza da ciência e a questão do gênero feminino na formação } \\
\text { docente/HEERDT, B. e BATISTA, I. L. }\end{array}$ & $\mathrm{T} 7$ \\
\hline ENPEC 2013 & Corpo, gênero e sexualidade no espaço escolar: lembranças de futuros/as professores/as/SANTOS, S. P. & T8 \\
\hline ENPEC 2013 & $\begin{array}{l}\text { Questões de gênero na ciência e na educação científica: uma discussão centrada no prêmio Nobel de física de } \\
\text { 1903/CORDEIRO, M. D. }\end{array}$ & T9 \\
\hline ENPEC 2013 & $\begin{array}{l}\text { Saberes docentes e invisibilidade feminina nas ciências/BATISTA, I. L.; HEERDT, B.; KIKUCHI, L. A.; CORREA, } \\
\text { M. L.; BARBOSA, R. G. e BASTOS, V. C. }\end{array}$ & $\mathrm{T} 10$ \\
\hline
\end{tabular}


Quadro 2: Trabalhos publicados em eventos nacionais entre 2015 e 2017

\begin{tabular}{|c|c|c|}
\hline Evento & Artigo/Autores & Código \\
\hline ENPEC 2015 & $\begin{array}{l}\text { Formação de professores no Brasil e questões de gênero feminino em atividades científicas/BATISTA, I. L.; SOUZA, } \\
\text { D. C.; KIKUCHI, L. A.; CORRÊA, M. L.; HEERDT, B.; STAL, J. Ç.; COSTA, M. e CHIARI, N. D. A. }\end{array}$ & T11 \\
\hline ENPEC 2015 & $\begin{array}{l}\text { Perspectiva de género y diversidad cultural en la enseñanza de las ciencias: mapeamiento informacional bibliográfico } \\
\text { (MIB)/ROJAS, Q. A. S. e ANDRADE, A. M. }\end{array}$ & T12 \\
\hline ENPEC 2015 & Pesquisas na área de educação científica a respeito de questões de gênero no Brasil/CHIARI, N. D. A. e BATISTA, I. L. & T13 \\
\hline ENPEC 2015 & Saberes docentes: natureza da ciência e as relações de gênero na educação científica/HEERDT, B. e BATISTA, I. L. & T14 \\
\hline ENPEC 2015 & Sexualidade e gênero na pauta escolar: mediações com a literatura paradidática/SILVA, A. C. e SIQUEIRA, V. H. F. & T15 \\
\hline ENPEC 2015 & $\begin{array}{l}\text { Articulando química, questões raciais e de gênero numa oficina sobre diversidade desenvolvida no âmbito do } \\
\text { PIBID: análise da contribuição dos recursos didáticos alternativos/SANTOS, R. G.; SIEMSEN, G. H. e SILVA, C. S. }\end{array}$ & T16 \\
\hline ANPED 2015 & Gênero e educação superior: um estudo sobre as mulheres na física/PINTO, E. J. S. e AMORIM, V. G. & T17 \\
\hline ANPED 2015 & $\begin{array}{l}\text { Discursos de bolsistas do programa institucional de bolsa de iniciação à docência sobre sexualidade/SILVA, E. P. } \\
\text { Q. e PARREIRA, F. L. D. }\end{array}$ & T18 \\
\hline ENPEC 2017 & $\begin{array}{l}\text { Desigualdades de gênero no contexto de um curso de licenciatura em ciências biológicas/CHIARI, N. D. A. e } \\
\text { BATISTA, I. L. }\end{array}$ & T19 \\
\hline ENPEC 2017 & É possível ser mulher na ciência?/ROSENTHAL, R. e REZENDE, D. B. & T20 \\
\hline ENPEC 2017 & Gênero: questão sociocientífica no ensino de ciências/MARTINS, L. G. e LOPES, N. & T21 \\
\hline ENPEC 2017 & O sexismo e suas consequências: um ensaio sobre a percepção de ciência/MARCHI, M. e RODRIGUES, A. & T22 \\
\hline ENPEC 2017 & $\begin{array}{l}\text { Questões de gênero na educação científica: tendências nas pesquisas nacionais e internacionais/SILVA, A. F.; } \\
\text { SANTOS, A. P. O. e HEERDT, B. }\end{array}$ & T23 \\
\hline ENPEC 2017 & Saberes docentes: mulheres na ciência/HEERDT, B. e BATISTA, I. L. & T24 \\
\hline ENPEC 2017 & A educação em ciências e a perspectiva de gênero/ALMEIDA, E. A. E. e FRANZOLIN, F. & T25 \\
\hline ENPEC 2017 & $\begin{array}{l}\text { Diversidade sexual, de gênero e raça/etnia nos trabalhos apresentados nas duas últimas edições do ENPEC } \\
\text { (encontro nacional de pesquisa em educação em ciências) (2013-2015)/GONTIJO, L. S.; SOARES, Z. M. P.; } \\
\text { GRACIANO, P. H. L. e FARIA, J. M. L. }\end{array}$ & T26 \\
\hline ENPEC 2017 & $\begin{array}{l}\text { Sexualidade, gênero e educação sexual nas atas do encontro nacional de pesquisa em educação em ciências - } \\
\text { ENPEC de } 2009 \text { a 2015/MIRANDA, P. R. M.; KALHIL, J. B. e ALVES, J. M. }\end{array}$ & T27 \\
\hline ANPED 2017 & $\begin{array}{l}\text { O ataque à discussão de gênero na escola, construção identitária e a importância da liberdade docente/MONTEIRO, } \\
\text { M. V. P. }\end{array}$ & T28 \\
\hline ANPED 2017 & $\begin{array}{l}\text { Amor, cuidado e competência: um olhar de gênero sobre a profissionalização do trabalho docente/SCHERER, } \\
\text { R. P. e DAL'IGNA, M. C. }\end{array}$ & T29 \\
\hline ANPED 2017 & $\begin{array}{l}\text { "Coisas de meninos e coisas de meninas": a produção do curso gênero e diversidade na escola sobre educação } \\
\text { infantil/SILVA, F. J. C. e MORAIS, A. B. A. }\end{array}$ & T30 \\
\hline ANPED 2017 & $\begin{array}{l}\text { Refletindo sobre gênero, trabalho e formação docente: um olhar para o pedagogo do gênero masculino iniciante } \\
\text { na educação infantil/CRUZ, S. P. S. e SOUSA, F. S. }\end{array}$ & T31 \\
\hline
\end{tabular}

UR 3. Gênero e estudos teóricos. Os artigos classificados nesse eixo apresentam uma revisão teórica sobre a necessidade da incorporação das questões de Gênero nas investigações em Educação em Ciências e Matemática (Batista et al., 2011, p. 5). UR 4. Gênero, práticas e Formação de Professores. Os artigos classificados nesse eixo analisam as noções e a Formação Docente acerca das questões de Gênero (Silva et al., 2017, p. 3). UR 5. Gênero e a participação de Mulheres na Ciência. Os artigos classificados nesse eixo analisam a participação das mulheres na História e na atualidade da Ciência (Silva et al., 2017, p. 3).

UR 6. Gênero e Intervenções escolares. Os artigos classificados nesse eixo apresentam abordagens metodológicas das questões de Gênero na Ciência em sala de aula (Silva et al., 2017, p. 3). UR 7. Gênero e Mídia. Os artigos classificados nesse eixo analisam como questões de Gênero relacionadas à área de Ciência são abordadas em diferentes mídias (Silva et al., 2017, p. 3).

\section{Resultados, Inferências e Interpretação}

Todos os trabalhos foram agrupados e classificados nas UR acima apresentadas e seguem expostos no Quadro 3. Esclarecemos que um trabalho pode ser classificado em mais de uma UR em função da abrangência das temáticas nele tratadas. Para nossa análise foram utilizados todos os trabalhos presentes 
Quadro 3: Número de trabalhos analisados com suas respectivas Unidades de Registro

\begin{tabular}{|c|c|c|}
\hline Unidades de Registro & $\begin{array}{c}\text { No. de } \\
\text { Trabalhos }\end{array}$ & Código dos Trabalhos \\
\hline 1. Gênero e materiais didáticos & 01 & T1 \\
2. Gênero e inferências de interpretação & 06 & T2; T3; T18; T19; T20; T25 \\
3. Gênero e estudos teóricos & 11 & T4; T5; T12; T13; T23; T26; T27; T28; T29; T30; T31 \\
4. Gênero, práticas e Formação de Professores & 16 & T5; T6; T7; T8; T10; T11; T12; T13; T14; T16; T17; T18; T19; T21; T22; T24 \\
5. Gênero e a participação de Mulheres na Ciência & 07 & T9; T10; T11; T17; T19; T20; T24 \\
6. Gênero e intervenções escolares & 02 & T15; T16 \\
7. Gênero e Ciência na Mídia & 01 & T22 \\
\hline
\end{tabular}

no Quadro 1 e no Quadro 2, totalizando 31 produções. Nesta seção, buscou-se uma análise com ampliação, somando os trabalhos já identificados por Batista et al. (2011) e Chiari e Batista (2015), com os identificados no intervalo estabelecido neste trabalho de 2015 a 2017.

Adotou-se a unitarização encontrada nos artigos de Batista et al. (2011) e por Silva et al. (2017) e as análises serão complementadas com a apresentação de um resumo de cada artigo e por fim um comparativo com as análises das pesquisas anteriores. A seguir, é retratado um quadro em que todos os trabalhos estão apresentados nas suas Unidades de Registro e a quantidade de trabalhos em cada UR.

Gênero e Materiais Didáticos: foi encontrado apenas um trabalho. O trabalho (T1) de Casagrande e Carvalho (2006) analisa as representações de Gênero de enunciados e ilustrações nos livros didáticos de Matemática para Educação Infantil. Os autores concluem que os padrões de lazer e de estudo, para as meninas é passivo e para os meninos é ativo, reproduzindo estereótipos. Esta UR apresenta que ainda há uma grande necessidade de estudos relacionados a Gênero e materiais didáticos e a pesquisa revela a falta de equidade entre os Gêneros.

Gênero e Inferências de Interpretação: Foram encontrados seis trabalhos. No artigo (T2) de Lima et al. (2007) o enfoque foi sociocultural. Por meio de questões abertas respondidas por alunas e alunos do Ensino Médio de três escolas diferentes, foram identificadas nas respostas dos meninos discursos que potencializam o acesso ao conhecimento científico, o que não foi encontrado nos discursos das meninas.

Sousa et al. (2007) (T3) analisaram relatos de alunos do Ensino Médio que participaram do Programa de Vocação Científica na Fundação Oswaldo Cruz de dois estados brasileiros. As características atribuídas às jovens meninas foram positivas, pois além de maturidade e organização, as participantes apresentaram interesse no futuro profissional. A pesquisa destacou também a Biologia como uma área vista como conhecimento feminino.

A pesquisa realizada (T18) por Silva e Parreira (2015) com alunos bolsistas do PIBID de Ciências Biológicas, identificou por meio de questionário, entrevista e grupo focal, discursos de hegemonia e de uma sexualidade fortemente baseada na Biologia.

Na investigação (T19) de Chiari e Batista (2017) as autoras buscam discutir desigualdades de Gênero com estudantes de licenciatura em Ciências Biológicas da UEL. A maioria dos estudantes identificaram situações de desigualdade de Gênero, nas quais meninas ou mulheres foram discriminadas por meninos ou homens no espaço acadêmico e principalmente por docentes.

O trabalho (T20) de Rosenthal e Rezende (2017) utiliza um software para evidenciar as palavras mais citadas nas entrevistas feitas com mulheres que estão no meio acadêmico. As questões que nortearam a pesquisa foram os fatores que têm contribuído para afastar as mulheres da carreira científica e quais têm auxiliado na permanência na carreira acadêmica.

Almeida e Franzolin (2017) (T25) investigam as concepções das meninas nos anos iniciais do Ensino Fundamental em relação à possibilidade das mulheres atuarem fazendo Ciência; analisaram o comportamento nas aulas de Ciências e quais assuntos relacionados a temas científicos elas se interessaram mais. Ao final da pesquisa, as meninas reconheceram mulheres como cientistas, entretanto, algumas afirmaram que tal carreira é para homens, poucas apresentaram interesse pela Ciência.

Por meio dessa Unidade de Registro é observada uma preocupação com a baixa participação das meninas/mulheres em temas relacionados à Ciência. Essa falta de interesse pode derivar de fatores como discriminação nas escolas e universidades e no âmbito familiar.

Gênero e Estudos Teóricos: foram encontrados 11 trabalhos. Lima e colaboradores (2009) (T4) realizaram um levantamento em periódicos em Ensino de Ciências no período de 2005 a 2007. O trabalho teve como objetivo investigar o efeito diferenciado da educação formal entre estudantes. Batista e colaboradores (2011) (T5) buscaram em artigos científicos e em atas de eventos no período de 2005 a 2011, trabalhos que discutissem Gênero na pesquisa em Formação de Professores em Educação em Ciências e Matemática no Brasil. 
As pesquisadoras Rojas e Andrade (2015) (T12) buscaram artigos em quatro revistas que relacionassem Gênero com atuação de professores de Ciências. Chiari e Batista (2015) (T13) realizam levantamento de publicações entre 2010 e 2014 em periódicos, atas de eventos e em dissertações e teses da área de Ensino de Ciências. Buscavam conhecer a atual produção científica brasileira a respeito de Gênero no Ensino de Ciências e na Formação de Professores da área. Silva et al. (2017) (T23) realizaram uma pesquisa em nível nacional e internacional em periódicos de estratos A1, A2 e B1 na área de Ensino. O levantamento foi feito no período de 2003 a 2016. A pesquisa aponta maior quantidade de trabalhos publicados em nível internacional, mostrando a necessidade de publicações em revistas nacionais.

Gontijo e colaboradores (2017) (T26) buscaram trabalhos apresentados na nona e décima edição do ENPEC, a fim de compreender como a pesquisa em Ciências se articula com diversidade sexual, Gênero e raça/etnia. Nesta mesma perspectiva, (T27) de Miranda et al. (2017) tinham como objetivo investigar como os temas de sexualidade, Gênero e educação sexual foram abordados nos trabalhos do ENPEC no período de 2009 a 2015.

A pesquisa (T28) de Monteiro (2017) apresenta uma reflexão entre as relações de construção igualitária, Gênero e interculturalidade, no sentido de limitar a liberdade dos professores na escola. Baseado no campo dos estudos culturais discute o que o cerceamento da liberdade docente implica na ação do professor e na educação de crianças.

No mesmo sentido, (T29) Scherer e Dal'Igna (2017) examinaram também o trabalho docente vinculado a processos de feminização e profissionalização do magistério; elas se pautaram nos estudos pós-estruturalistas. Observaram que na literatura brasileira ocorreu um deslocamento nos anos 1980 da feminização do magistério e atualmente as professoras têm mobilizado saberes para defender um compromisso político com a escola e os conhecimentos escolares.

Silva e Moraes (2017) (T30) analisaram as relações de Gênero nas instituições de Educação Infantil em diversas cidades por meio de onze trabalhos de conclusão de curso de Especialização em Gênero e Diversidade na Escola. O trabalho (T31) de Cruz e Sousa (2017) analisa produções depositadas no Banco de Teses e Dissertações do Instituto Brasileiro de Informação em Ciência e Tecnologia, no período de janeiro de 2006 a março de 2017. Os dois trabalhos defendem a importância de estudos de Gênero na Formação de Professores e buscaram analisar a escola como espaço de discussão e de grandes interferências das relações de Gênero.

Os levantamentos realizados são importantes, pois apresentam tanto no âmbito nacional como internacional o panorama das pesquisas de Gênero na educação em Ciências. Por meio dessas pesquisas observa-se que há carência de conhecimentos desses temas, principalmente na formação inicial e continuada dos docentes. Existem lacunas também nos estudos das questões de Gênero no Ensino de Ciências.
Gênero, Práticas e Formação de Professores: foram encontrados 16 trabalhos. A pesquisa (T8) de Santos (2013) investigou narrativas de 23 professores a respeito de lembranças mais marcantes relacionadas ao corpo, Gênero e sexualidade. As narrativas foram construídas em um curso de Formação de Professores em Ciências Biológicas. Ele conclui que o espaço escolar produz e propaga ideais de masculino, feminino, desqualificação da homossexualidade e travestilidade.

Heerdt e Batista (2011) (T7) buscaram apresentar como as questões de Gênero podem se articular de maneira contextualizada, com a História e Filosofia da Ciência e a Natureza da Ciência. A discussão apresentou a relevância em desenvolver abordagens de inserção dos estudos de Gênero na Formação Docente.

Batista et al. (2011) (T5) analisaram como as pesquisas de Gênero feminino têm sido discutidas na Formação de Professores em Educação em Ciências e Matemática no Brasil. Essa análise se fez por meio de um levantamento realizado pelas autoras e autores e foram encontrados apenas sete artigos. Batista et al. (2013) (T10), em um curso de Formação Docente, investigaram noções e saberes de professores da região norte do Paraná a respeito da visibilidade da mulher na Ciência. E o resultado foi uma carência predominante do conhecimento do papel e da participação feminina na História da Ciência e na Formação Docente dos participantes.

Os mesmos pesquisadores apresentaram um trabalho no ENPEC de 2015 (T11) investigando as noções e os saberes de professores a respeito de Gênero no ambiente escolar. Nesta pesquisa foram coletadas informações de docentes dos estados do Paraná, Rio Grande do Norte, Rio de Janeiro, Belo Horizonte, Belém e Mato Grosso. O resultado reforça a escassez de conhecimentos dos temas abordados e a necessidade de se tratar, com enfoque metodológico adequado, tal tema na Formação Docente.

Lima e Siqueira (2011) (T6) investigaram os significados de professores de uma escola técnica do Rio de Janeiro diante de questões de Gênero e sexualidade. O enfoque biológico e adiantamento destas questões no debate em sala de aula foram predominantes nos discursos dos docentes.

Em um levantamento feito por Rojas e Andrade (2015) (T12), em quatro revistas que relacionassem Gênero com atuação de professores de Ciências, identificou-se que de 150 artigos encontrados, apenas $6 \%$ deles discutiam a temática, indicando um campo que ainda pode ser explorado, principalmente a respeito do que pensam os docentes.

O trabalho (T13) de Chiari e Batista (2015) se classifica nesta UR também, pois, por meio de um levantamento feito entre 2010 e 2014, as autoras buscaram conhecer a produção científica brasileira a respeito de Gênero no Ensino de Ciências e na Formação de Professores da área. Elas observaram um início de tendência de abordagens que incluem na formação inicial e em serviço de professores de Ciências a temática Gênero. 
Em uma pesquisa (T14) de Heerdt e Batista (2015) a respeito de saberes docentes, Natureza da Ciência e Gênero, as autoras buscaram compreender e explicitar tais saberes interligados com a Ciência, para isto, analisaram respostas de duas questões obtidas em um curso de Formação Docente. Por meio do curso e das respostas, pode-se perceber que os docentes refletiram a respeito das questões de Gênero e da Natureza da Ciência, além de ter possibilitado a construção de tais saberes docentes.

Heerdt e Batista (2017) (T24) buscaram explicitar alguns saberes docentes em relação a contribuição científica da mulher na Ciência e analisaram, por meio de planos de aulas, como tais saberes fazem parte dos seus planejamentos pedagógicos. Destacaram a falta de conhecimento da participação feminina na História da Ciência.

O trabalho (T16) de Santos et al. (2015) foi desenvolvido por alunos do PIBID em que eles elaboraram e aplicaram uma oficina por meio de recursos alternativos a fim de discutir conceitos químicos, questões raciais e Gênero. As autoras concluíram que houve essa articulação entre os temas, proporcionando a contribuição para o Ensino de Ciências/ Química.

Nessa mesma linha, (T17) Pinto e Amorim (2015) examinaram as experiências acadêmicas de cinco mulheres estudantes do curso de Física. Apresentaram uma cultura preconceituosa e discriminatória por parte de seus colegas e professores da universidade. As estudantes relataram a pressão que existe em ter que se destacar mais do que os homens para serem aceitas no universo acadêmico.

Uma pesquisa realizada (T18) por Silva e Parreira (2015), com alunos bolsistas do PIBID de Ciências Biológicas, debateu a respeito de sexualidade com a futura atuação docente. Foram analisados os discursos dos bolsistas relacionados a diferentes espaços em que foram apontados múltiplos discursos.

Chiari e Batista (2017) (T19), por meio de uma abordagem didática aplicada a alunos de Ciências Biológicas da UEL, discutiram questões de Gênero na formação inicial dos futuros docentes atrelando a Educação Científica. Os pesquisados reconheceram a desigualdade entre homens e mulheres existente no ambiente educacional.

O trabalho (T21) de Martins e Lopes (2017) analisou duas oficinas com alunos e docentes da área de Ciências a fim de identificar as potencialidades e as dificuldades das abordagens das questões sociocientíficas relacionadas a Gênero no Ensino de Ciências. Os pesquisadores identificaram a necessidade de se debater tal temática, para esclarecer equívocos conceituais e controvérsias construídas ligadas com a palavra Gênero.

Por fim, Marchi e Rodrigues (2017) (T22), com um recurso midiático, analisaram comentários agressivos e discriminatórios de professores, com o objetivo de identificar como tais comentários desmotivam estudantes mulheres do Instituto de Física da USP.
Um destaque é que dentre os 16 trabalhos apresentados, oito são trabalhos originados do grupo Investigações em Filosofia e História da Ciência e Educação em Ciências e Matemática, apresentando a preocupação do grupo em desenvolver intervenções.

Gênero e Participação de Mulheres na Ciência: foram encontrados sete trabalhos. Cordeiro (2013) (T9) explorou o prêmio Nobel de Física de 1903, destacando a participação de Marie Curie, suas potencialidades e dificuldades, além de discutir na Formação Docente os preconceitos que ainda existem na Ciência. Essa pesquisa evidencia a relevância da participação feminina para a contribuição da Ciência.

Os trabalhos (T10) e (T11) de Batista et al. (2013; 2015) também são classificados nesta UR, pois foram investigadas noções e saberes dos professores de 5 estados brasileiros diferentes a respeito da visibilidade da mulher na Ciência, evidenciando a necessidade de se conhecer melhor o papel feminino na História da Ciência e na Formação Docente dos participantes.

Pinto e Amorim (2015) (T17) entrevistaram estudantes do curso de Física e apontaram que ainda há uma cultura de preconceito e discriminação na academia. O que gera desconforto, desânimo e pressão para serem aceitas, levandoas a se destacarem mais do que os homens para obterem reconhecimento profissional.

As pesquisadoras Chiari e Batista (2017) (T19) desenvolveram uma abordagem didática aplicada a alunos de Ciências Biológicas da UEL, em que foram discutidos alguns temas de desigualdade de Gênero, entre eles, a discriminação das mulheres, privilégio de homens, relações de poder e igualdade de tratamento no meio acadêmico.

Com o uso de um software, Rosenthal e Rezende (2017) (T20) evidenciam as palavras mais citadas nas entrevistas feitas com mulheres que estão no meio acadêmico. Analisam os fatores que têm contribuído para a participação e para o afastamento das mulheres na Ciência.

Na intervenção pedagógica (T24) realizada com docentes da Educação Básica que atuam na área de Ciências Biológicas no Paraná, Heerdt e Batista (2017) buscaram saberes docentes em relação à contribuição científica da mulher na Ciência. Por meio dessa pesquisa, percebeu-se a necessidade de docentes buscarem mais conhecimento na História da Ciência para reconhecerem e disseminarem a participação de mulheres na Ciência.

Por meio desta UR identifica-se que o tema Gênero e participação de mulheres na Ciência são discussões recentes, pois os primeiros trabalhos encontrados nesses eventos em que é pesquisada diretamente esta temática são os de Cordeiro (2013) e de Batista et al. (2013). Desde então, as discussões e o destaque favorecendo a participação feminina na Ciência têm aumentado, porém com uma frequência ainda baixa.

Gênero e Intervenções Escolares: foram encontrados dois trabalhos. O trabalho (T15) de Silva e Siqueira (2015) analisou 
livros paradidáticos como recurso de discussão de questões de sexualidade e Gênero com docentes e com grupos focais de alunos em quatro escolas do Ensino Fundamental do Rio de Janeiro. Outro trabalho (T16) foi de Santos et al. (2015), as autoras desenvolveram uma oficina a respeito de diversidade com alunos do PIBID articulando Química, questões raciais e de Gênero. As contribuições de tal oficina foram muitas, e destaca-se a possibilidade de trabalhar conceitos químicos com o tema diversidade utilizando recursos didáticos alternativos.

Há poucos trabalhos apresentados nesses eventos que relatam intervenções realizadas nas escolas, principalmente relacionados a disciplinas científicas. Porém, se percebe que é possível desenvolver tais práticas.

Gênero e Ciência na Mídia: foi encontrado um trabalho. Marchi e Rodrigues (2017) (T22) destacaram relatos de estudantes mulheres do Instituto de Física da USP obtidos por meio de uma página do Facebook. O objetivo foi procurar entender de que forma os comentários e concepções dos professores podem contribuir no compreender o que é Ciência e no caráter agressivo e discriminatório que os relatos apresentaram. É interessante analisar o uso de um recurso midiático para o debate e relatos das estudantes quando são discriminadas ou pré-julgadas. Outro aspecto é a área de formação das estudantes (Física), uma área predominantemente masculina que ainda se requer grandes reconhecimentos da participação feminina.

\section{Metatexto}

De acordo com o referencial seguido (Bardin, 2004), para uma inferência dedutiva e interpretação dos resultados é proposto um texto logo em seguida da unitarização e descrição dos dados. Esse texto dialoga com os referenciais teóricos que sustentaram esta pesquisa e as Unidades de Contexto e de Registros.

Destaca-se que as Unidades de Registro prévias foram baseadas no trabalho de Batista et al. (2011) que realizou um levantamento nos anos de 2005 a 2011 em periódicos da área de Ensino de Ciências e Matemática e nas atas dos eventos ANPED e ENPEC, pesquisando a temática Gênero na Educação Científica e na Formação Docente. Todos os trabalhos encontrados foram unitizados em três eixos: "Gênero e materiais didáticos", "Gênero e inferências de interpretação" e "Gênero e estudos teóricos", e foi identificado que não havia publicações no eixo de Formação de Professores em Ciências da Natureza e Matemática, mostrando a premente necessidade de pesquisas nessas temáticas.

O segundo referencial utilizado na unitarização desta investigação é o trabalho de Silva et al. (2017), que buscaram artigos em revistas nacionais e internacionais online no Ensino de Ciências entre os anos de 2003 a 2016. As autoras utilizaram os três eixos de Batista et al. (2011) e elaboraram cinco novas
Unidades de Registro. Sendo que dessas cinco, quatro delas foram utilizadas para a análise deste trabalho.

Na primeira UR - Gênero e materiais didáticos, foi encontrado apenas um trabalho que analisa as representações de Gênero nos livros didáticos, publicado em 2006 no ANPED. Este trabalho (T1) é o mesmo identificado por Batista et al. (2011), que no total encontraram dois artigos, sendo o outro publicado em periódico. Para esta mesma UR, Silva et al. (2017) encontraram também dois artigos, sendo um deles em revista internacional e o outro foi o mesmo trabalho identificado por Batista et al. (2011).

Destacam-se as datas de publicações desses trabalhos nos eventos ANPED e ENPEC e nas revistas investigadas, entre os anos de 2006 a 2008, ou seja, faz 10 anos que não são publicados trabalhos que investigam Gênero nos livros didáticos, tanto no âmbito nacional como no internacional na área de Ensino de Ciências. Isto é preocupante, pois Fiorese e Delizoicov (2015) apresentam que o livro didático continua sendo o principal recurso utilizado pelo professor no processo de ensino e de aprendizagem em sala de aula, visto que na Educação Pública do Brasil o livro didático é financiado pelo Governo e fornecido a todos os alunos.

Por meio da investigação de Casagrande e Carvalho (2006) foram identificados padrões estereotipados para meninas e meninos, logo, houve a provável reprodução e a propagação desses padrões por meio do livro didático para crianças desde o início da vida escolar.

Na segunda UR - Gênero e Inferências de Interpretação foram identificados seis trabalhos, a mesma quantidade de artigos que foram encontrados em revistas na pesquisa de Silva et al. (2017). Dois dos trabalhos identificados nas atas dos eventos desta pesquisa também foram classificados por Batista et al. (2011).

Nessa UR os trabalhos apresentam inferências da representação da mulher nas Ciências, bem como as desigualdades de Gênero e a influência de tal desigualdade como causa de discriminação e desmotivação de mulheres para seguirem carreira nas Ciências da Natureza e Engenharias. Destaca-se que investigações da sub-representação da mulher ocorrem nos anos iniciais do Ensino Fundamental (T24), no Ensino Médio (T2; T3) e no Ensino Superior (T18; T19 e T20).

Tindall e Hamil (2004) discutiram a respeito da influência que a sociedade, a família e a escola têm na escolha da carreira profissional de meninos e meninas. Por meio dos resultados desta investigação, se observa que a invisibilidade da mulher cientista e a desmotivação por áreas de Ciências aplicadas ou da Natureza inicia-se desde os primeiros anos escolares e quando se chega à universidade essa desigualdade reflete na permanência das mulheres no campo científico.

Na terceira UR - Gênero e estudos teóricos foram encontrados 11 trabalhos que por meio dos levantamentos apresentam a carência de conhecimentos e de publicações relacionadas à temática Gênero no Ensino de Ciências. Entre 
os anos de 2005 a 2011 Batista et al. (2011) encontraram apenas dois trabalhos que se encaixaram nesta UR, sendo um deles publicado em revista e o outro publicado no ENPEC. Na pesquisa de nível nacional e internacional, realizada por Silva et al. (2017), foram encontrados sete artigos, destes, apenas um é nacional.

Os trabalhos dessa UR apresentam levantamentos em revistas nacionais e internacionais classificados no Qualis da CAPES da área de Ensino e Educação nos estratos A1, A2, B1 e B2, em atas de eventos, dissertações e teses da área de Ensino de Ciências e Matemática, na formação e na ação docente. Por meio dessas pesquisas pode-se obter um panorama das discussões de Gênero no âmbito escolar e, como visto, para a necessidade de incorporar as questões de Gênero na Educação em Ciências e Matemática, bem como em novas pesquisas da área.

A partir do mapeamento da situação atual das pesquisas de Gênero, o passo seguinte é estudar teoricamente outros aspectos como sala de aula, ação dos professores, destaques de participação feminina na Ciência, materiais didáticos, ou seja, há campo expandido de novas pesquisas que abordem outros temas, uma vez que por meio do Quadro 3 se deduz que há um reduzido número de trabalhos resultantes de pesquisas nessa temática.

A UR 4 - Gênero, práticas e Formação de Professores é a UR com maior quantidade de trabalhos identificados, no total foram 16. Na pesquisa de Silva et al. (2017) foram encontrados cinco trabalhos, sendo 3 deles internacionais.

Um destaque para esta UR é a relevância de se construir abordagens para a discussão desta temática, pois, por meio desses trabalhos, se reconhece a discriminação e o preconceito que docentes ainda encontram na formação inicial e em serviço. Por meio dos levantamentos na UR 3 se identifica que os docentes e futuros docentes estão despreparados para tratar com as situações que relacionam Gênero e a Educação em Ciências.

Mesmo encontrando 16 trabalhos que apresentam abordagens e que forneçam algum tipo de momento de reflexão e formação para os professores, ainda há a necessidade de elaborar cursos e momentos de formação inicial e em serviço, que de forma prática forneçam instrumentos para que docentes apliquem seus conhecimentos em sala de aula, independente dos níveis de ensino.

Ressalte-se que todas as pesquisas mostraram a falta de conhecimento dos docentes em relação à participação feminina na Ciência e das discussões de Gênero, a existência de preconceito e discriminação no ambiente educacional e a predominância biológica nas discussões de Gênero. Assuntos que por meio de Formação Docente são discutidos e possibilitam a desconstrução de estereótipos nos professores e a não propagação destes estereótipos em sala de aula.

Na UR 5 - Gênero e participação de mulheres na Ciência, foram encontrados sete trabalhos nesta Unidade, sendo que as primeiras publicações foram a partir de 2013 (T9 e T10). Esta
UR analisa trabalhos que discutem a participação feminina na Ciência tanto na história como também na atualidade. Identifica-se que apenas Cordeiro (2013) (T9) pesquisa a participação de uma cientista na história, analisando o Prêmio Nobel de Física de 1903 destinado a Marie Sklodowska Curie e Heerdt e Batista (2017) (T24) que utilizaram da História da Ciência para discutir a participação feminina em uma abordagem didática para docentes. Os outros cinco trabalhos investigam a participação feminina na Formação Docente com participações na atualidade, tanto com professores quanto com estudantes.

$\mathrm{Na}$ investigação de Silva et al. (2017) foram encontrados três artigos, dois em revistas internacionais que tratavam da participação das mulheres ao longo da História da Ciência e um nacional que pesquisou trajetórias de mulheres cientistas universitárias.

Assim como na UR 4, os trabalhos relatavam falta de conhecimento das questões de Gênero, nesta UR 5 isto se confirma por meio de poucas pesquisas que investigam a participação de mulheres na Ciência. A participação feminina nos assuntos científicos sempre foi presente, porém, nunca reconhecida pelos cientistas masculinos, isso gerou invisibilidade da contribuição da mulher, principalmente nas áreas de Ciências da Natureza.

A UR 6 - Gênero e Intervenções escolares foram identificados dois trabalhos, sendo que (T15) trabalhou com grupo de docentes e alunos de quatro escolas e analisou as questões de Gênero por meio do uso de livros paradidáticos e no (T16) foram desenvolvidas atividades por meio de uma oficina de Química relacionando Gênero e questões raciais. Identifica-se uma baixa preocupação em levar até a escola a discussão de temas relacionados a Gênero. Observa-se que não há trabalhos publicados que apresentem intervenções para o Ensino Superior, apontando uma lacuna neste nível de ensino.

Por meio da UR 2 se observa que, quando questionados, os docentes sentem diferenças no tratamento e nos discursos dos alunos, o que reforça a necessidade de elaborar abordagens e intervenções que gerem, na prática de sala de aula, discussões da temática Gênero com os conteúdos científicos.

No levantamento em revistas de Silva et al. (2017) também foram encontrados dois artigos, um que realiza uma intervenção em uma escola secundária sueca e o outro apresenta uma proposta para o Ensino Superior em Química.

Na última UR 7 - Gênero e Ciência na Mídia foi encontrado apenas um trabalho (T22) que buscou em páginas de uma rede social muito utilizada por brasileiros, relatos de estudantes de um curso de Física em que a predominância é masculina, quais influências que docentes têm na discriminação das estudantes. Em periódicos da área de Ensino de Ciências, a publicação de trabalhos que discutem essa UR também é pouco presente. De acordo com Silva et al. (2017), foram encontrados apenas três artigos, sendo que apenas um deles é publicado em revista brasileira. 
Utilizar recursos midiáticos para investigação na área de Gênero é pequena. Entretanto, a mídia em geral transmite imagens estereotipadas de cientistas, assim se não houver a sensibilização dos docentes para que reconheçam e desfaçam esses estereótipos, é improvável que trabalhem com seus alunos esta temática.

\section{Conclusões}

Neste trabalho buscamos evidenciar a inserção da temática de Gênero na Formação Docente de Ciências, focalizando na visibilidade do Gênero feminino e sua participação ao longo da História da produção científica.

Os trabalhos identificados e analisados neste artigo evidenciam alguns aspectos acerca das pesquisas de Gênero na Formação Docente de Ciências da Natureza. Diante da análise realizada observa-se que uma maioria numérica dos trabalhos trata das questões de Gênero articuladas à prática docente, mas no aspecto majoritariamente de diagnósticos. Identificamos artigos de revisão teórica acerca da incorporação das questões de Gênero; sete trabalhos que versam a respeito da participação das mulheres na História da Ciência; seis trabalhos analisados estudam os motivos da frequência relativa do Gênero feminino e suas relações com a Ciência em cursos de diferentes níveis de ensino; apenas dois trabalhos apresentam abordagens metodológicas para serem desenvolvidas em sala de aula e, por fim, apenas um trabalho aborda as representações de Gênero nos livros didáticos e outro a respeito de questões de Gênero em diferentes mídias. Ao comparar esta pesquisa com a de Silva et al. (2017) percebe-se um resultado semelhante nos períodos e fontes de levantamento coincidentes.

Assim como a pesquisa das autoras acima mencionadas, esta investigação reconhece a necessidade de novas pesquisas que abordem a temática em foco, nas mais diferentes perspectivas. Dos resultados encontrados, destaca-se a pouca produção de pesquisas no desenvolvimento de abordagens metodológicas apropriadas a temática. Salientamos que o próximo objetivo é poder contribuir com um levantamento a ser realizado em dissertações e teses de programas de pós-graduação no Brasil, a fim de obtermos um panorama de como está sendo discutido Gênero na Ciência e na Formação Docente.

\section{Referências}

BARDIN, L. Análise de conteúdo. Lisboa: Ed. 70, 2004.

BATISTA, I. L.; HEERDT, B.; KIKUCH, L. A.; CORRÊA, M.
L.; BARBOSA, R. G. e BASTOS, V. C. Saberes docentes e invisibilidade feminina nas ciências. In: Anais do IX Encontro Nacional de Pesquisa em Educação em Ciências. Águas de Lindóia, SP, 2013.

; TOREJANI, A. T. C.; HEERDT, B.; LUCAS, L. B.; OHIRA, M. A.; CORRÊA, M. L.; BARBOSA, R. G. e BASTOS, V. C. Gênero feminino e formação de professores na pesquisa em educação científica e matemática no Brasil. In: Anais do VIII Encontro Nacional de Pesquisa em Educação em Ciências. Campinas, SP, 2011.

BOGDAN, R. e BIKLEN, S. Investigação qualitativa em educação: uma introdução à teoria e aos métodos. Porto: Porto Editora, 1994.

CHIARI, N. D. A. e BATISTA, I. L. Pesquisas na área de educação científica a respeito das questões de gênero no Brasil. In: Anais do X Encontro Nacional de Pesquisa em Educação em Ciências. Águas de Lindóia, SP, 2015.

FIORESE, J. Z. e DELIZOICOV, N. C. Livros didáticos de biologia e a história da ciência. Roteiro, v. 40, n. 1, p. 101-126, 2015.

HARDING, S. e MCGREGOR, E. The gender dimension of science and technology. In: MOORE, H. J. (Ed.). UNESCO world science report. Paris: UNESCO, 1996.

IFHIECEM. Investigações em filosofia e história da ciência, educação em ciências e matemática. Disponível em http://www.uel.br/grupopesquisa/ifhiecem/index.html, acessado em Fevereiro 2019.

LOPES, M. M. "Aventureiras" nas ciências: refletindo sobre gênero e história das ciências naturais no Brasil. Cadernos Pagu, n. 10, p. 345-368, 1998.

MATTHEWS, M. História, filosofia e ensino de ciências: tendência atual de reaproximação. Caderno Catarinense de Ensino de Física, v. 12, n. 3, p. 164-214, 1995.

MELO, H. P.; LASTRES, H. M. M. e MARQUES, T. C. N. Gênero no sistema de ciência, tecnologia e inovação no Brasil. Gênero, v. 4, n. 2, p. 73-94, 2004.

REZENDE, F. e OSTERMANN, F. A questão de gênero no ensino de ciências sob o enfoque sociocultural. In: Anais do XVII Simpósio Nacional de Ensino de Física. São Luís, MA, 2007.

ROSSI, A. Women in science: why so few? Science, v. 148, n. 3674, p. 1196-1202, 1965.

SILVA, A. F.; SANTOS, A. P. O. e HEERDT, B. Questões de gênero na educação científica: tendências nas pesquisas nacionais e internacionais. In: Anais do XI Encontro Nacional de Pesquisa em Educação em Ciências. Florianópolis, SC, 2017.

SOUZA, A. M. F. L. Ensino de ciências: onde está o gênero? Faced, n. 13, p. 149-160, 2008.

TINDALL, T. e HAMIL, D. B. Gender disparity in science education: the causes, consequences, and solutions. Education, v. 125, n. 2, p. 282-295, 2004. 